\title{
WEIGHTED POINCARÉ INEQUALITIES ON CONVEX DOMAINS
}

\author{
Seng-Kee Chua And Richard L. Wheeden
}

Abstract. Let $\Omega$ be a bounded open convex set in $\mathbb{R}^{n}$. Suppose that $\alpha \geq 0, \beta \in \mathbb{R}$, $1 \leq p \leq q<\infty$, and

$$
1-\frac{n}{p}+\frac{n}{q}, 1-\frac{n+\beta}{p}+\frac{n+\alpha}{q} \geq 0 .
$$

Let $\rho(x)=\operatorname{dist}\left(x, \Omega^{c}\right)=\min \left\{|x-y|: y \in \Omega^{c}\right\}$ denote the Euclidean distance to the complement of $\Omega$. Define $\rho^{\alpha}(\Omega)=\int_{\Omega} \rho(x)^{\alpha} d x$, and denote

$$
f_{\Omega, \rho^{\alpha}}=\frac{1}{\rho^{\alpha}(\Omega)} \int_{\Omega} f(x) \rho(x)^{\alpha} d x \quad \text { and } \quad\|f\|_{L_{\rho^{\alpha}}^{p}(\Omega)}=\left(\int_{\Omega}|f(x)|^{p} \rho(x)^{\alpha} d x\right)^{\frac{1}{p}} .
$$

We derive the following weighted Poincaré inequality for locally Lipschitz continuous functions $f$ on $\Omega$ :

$$
\left\|f-f_{\Omega, \rho^{\alpha}}\right\|_{L_{\rho^{\alpha}}^{q}(\Omega)} \leq C \eta^{\frac{\beta}{p}-\frac{\alpha}{q}}|\Omega|^{\frac{1}{q}-\frac{1}{p}} \operatorname{diam}(\Omega)^{1-\frac{\beta}{p}+\frac{\alpha}{q}}\|\nabla f\|_{L_{\rho^{\beta}}^{p}(\Omega)},
$$

where $\eta$ is the eccentricity of $\Omega$ and $C$ is a constant depending only on $p, q, \alpha, \beta$ and the dimension $n$. The main point of the estimate is the way the constant depends on $\eta$ for a general convex domain. We also consider the case $1 \leq q<p<\infty$, where the inequality is valid under the stronger hypothesis $1-(1+\beta) / p+(1+\alpha) / q>0$. When $q \geq p$, the case of convex domains which are symmetric with respect to a point was settled in [CD], and our estimate for $q \geq p$ extends that result to nonsymmetric domains. Moreover, the exponent of $\eta$ is sharp and the conditions are necessary.

\section{Introduction}

Let $\Omega$ be a bounded convex domain (i.e., a bounded open convex set) in $\mathbb{R}^{n}$. If $1 \leq p, q<\infty$ and $1 / q \geq 1 / p-1 / n$, it is well-known that the Poincaré inequality

$$
\left\|f-f_{\Omega}\right\|_{L^{q}(\Omega)} \leq C(p, q, n, \Omega)\|\nabla f\|_{L^{p}(\Omega)}, \text { with } f_{\Omega}=\frac{1}{|\Omega|} \int_{\Omega} f,
$$

holds for all Lipschitz continuous functions $f$ on $\Omega$. When $1 \leq q \leq p$, it was only realized recently in Acosta and Durán $[\mathrm{AD}]$ and in Chua and Wheeden [CW3] that the constant $C(p, q, n, \Omega)$ can be expressed as $C(p, q)|\Omega|^{1 / q-1 / p} \operatorname{diam}(\Omega)$. In particular, it is independent of both the eccentricity $\eta$ of $\Omega$ and the dimension $n$; see also [DL]. The precise definition of $\eta$ and some related discussion are given below.

In fact, when $1 \leq q \leq p$, Chua and Wheeden [CW3, Theorems 1.1 and 1.2] showed that for any $\alpha \geq 0$ and $f \in \operatorname{Lip}(\Omega)$,

$$
\left\|f-f_{\Omega, \rho^{\alpha}}\right\|_{L_{\rho^{\alpha}}^{q}(\Omega)} \leq C(p, q)\left(\int_{\Omega} \rho^{\alpha}\right)^{\frac{1}{q}-\frac{1}{p}} \operatorname{diam}(\Omega)\|\nabla f\|_{L_{\rho^{\alpha}}^{p}(\Omega)}
$$

Received by the editors March 25, 2010.

Key words and phrases. Poincaré inequalities, convex domains, John domains, Boman domains, eccentricity, distance weights, doubling measures. 
using the fact that $\rho$ is a concave function on $\Omega$; see [CW3, Remark 1.5(4)]. Moreover, since bounded convex sets are John domains, it follows from a generalization of the result in [C93, Th 1.5] or the proof of Theorem 1.3 in [HS] that if $\frac{1}{p} \geq \frac{1}{q} \geq \frac{1}{p}-\frac{1}{n}$, $1-\frac{n+\beta}{p}+\frac{n+\alpha}{q} \geq 0$ and $\alpha \geq 0$, then

$$
\left\|f-f_{\Omega, \rho^{\alpha}}\right\|_{L_{\rho^{\alpha}}^{q}(\Omega)} \leq C(p, q, \alpha, \beta, n, \eta)|\Omega|^{\frac{1}{q}-\frac{1}{p}} \operatorname{diam}(\Omega)^{1-\frac{\beta}{p}+\frac{\alpha}{q}}\|\nabla f\|_{L_{\rho^{\beta}}^{p}(\Omega)} .
$$

If $\Omega$ is also symmetric with respect to a point, it has been shown recently in [CD] that the constant $C(p, q, \alpha, \beta, n, \eta)$ in $(1.2)$ is at most $C(p, q, \alpha, \beta, n) \eta^{\frac{\beta}{p}-\frac{\alpha}{q}}$, with the same restrictions on the parameters. A main goal of the present paper is to extend this fact to all bounded convex domains. The principal difficulty in doing so is finding a natural metric to work with. Moreover, nonsymmetric convex domains have no center in the usual sense. However, the key ideas we will use are simple. We will identify the collection of all translates and (suitable) dilates of $\Omega$ with the collection of balls in a naturally associated space of homogeneous type, and then apply Poincaré estimates from [CWa], [CWb]. Thus, instead of covering $\Omega$ by Euclidean Whitney balls or cubes, we will cover it by $\delta$-Whitney $\Phi$-balls (see Definition 2.1 ) and note that $\Omega$ is then a Boman domain (hence a John domain) with parameter independent of the eccentricity. If one instead covers $\Omega$ by Euclidean balls or cubes, then even though it is still a Boman domain, the corresponding parameter will unfortunately depend on the eccentricity. Another goal of the paper is to derive estimates in case $1 \leq q<p<\infty$. We also consider functions $f$ which are only locally Lipschitz continuous, while [CD] requires global Lipschitz continuity.

Before we state our main result, let us define the notion of eccentricity that we will use. We begin by listing a fact [W, Theorem 7.1.5] which is a consequence of Helly's theorem: For every bounded convex set $\Omega$ in $\mathbb{R}^{n}$, there is a point $x^{o} \in \Omega$ and a constant $\nu$ with $1 \leq \nu \leq n$ such that

$$
k\left(x^{o}-x\right)+x^{o} \in \Omega \text { for all } x \in \Omega \text { and all } k \in[0,1 / \nu] .
$$

With $\Omega$ fixed and $x^{o}$ chosen in this way, we will refer to $x^{o}$ as the center of $\Omega$. Let $\Omega-x^{o}$ denote the image of $\Omega$ under the translation $x \rightarrow x-x^{o}$ of $\mathbb{R}^{n}$ which maps $x^{o}$ to the origin:

$$
\Omega-x^{o}=\left\{x-x^{o}: x \in \Omega\right\} .
$$

Let $\rho_{o}$ denote the Euclidean distance from $x^{o}$ to $\partial \Omega$ (equivalently, the distance from 0 to $\left.\partial\left(\Omega-x^{o}\right)\right)$ :

$$
\rho_{o}=\rho\left(x^{o}\right)=\min \left\{\left|x-x^{o}\right|: x \in \partial \Omega\right\}=\min \left\{|x|: x \in \partial\left(\Omega-x^{o}\right)\right\} .
$$

Then by (4.3) in the Appendix,

$$
\max _{x \in \Omega} \min _{y \in \partial \Omega}|x-y| \leq(1+\nu) \rho_{0} .
$$

We define the eccentricity $\eta$ of $\Omega$ by

$$
\eta=\max \left\{\left|x-x^{o}\right|: x \in \partial \Omega\right\} / \min \left\{\left|x-x^{o}\right|: x \in \partial \Omega\right\}=\frac{1}{\rho_{o}} \max \left\{\left|x-x^{o}\right|: x \in \partial \Omega\right\} .
$$


While this definition of eccentricity depends on $x^{o}$ and is different from the classical one, the two are equivalent in size, with constants of equivalence that are independent of $\Omega$ (see (4.1) and (4.2) in the Appendix).

The following is our main result.

Theorem 1.1. Let $\Omega \subset \mathbb{R}^{n}$ be a bounded convex domain with eccentricity $\eta$. Let $1 \leq p, q<\infty$ satisfy $1 / q \geq 1 / p-1 / n$, and suppose $\alpha \geq 0$ and $\beta \in \mathbb{R}$. Denote $\rho(x)=\min \left\{|x-y|: y \in \Omega^{c}\right\}$ and define $\rho^{a}(\Omega), f_{\Omega, \rho^{a}}$ and $\|\cdot\|_{L_{\rho^{a}}^{p}(\Omega)}$ as in the Abstract for $a=\alpha$ or $\beta$.

(i) If $p \leq q$ and $1-\frac{n+\beta}{p}+\frac{n+\alpha}{q} \geq 0$, then there is a constant $C$ depending only on $p, q, \alpha, \beta$ and $n$ such that for all locally Lipschitz continuous functions $f$ on $\Omega$,

$$
\left\|f-f_{\Omega, \rho^{\alpha}}\right\|_{L_{\rho^{\alpha}}^{q}(\Omega)} \leq C \eta^{\frac{\beta}{p}-\frac{\alpha}{q}}|\Omega|^{\frac{1}{q}-\frac{1}{p}} \operatorname{diam}(\Omega)^{1-\frac{\beta}{p}+\frac{\alpha}{q}}\|\nabla f\|_{L_{\rho^{\beta}}^{p}(\Omega)} .
$$

(ii) If $q<p$, then (1.8) is true if $1-(1+\beta) / p+(1+\alpha) / q>0$.

In Theorem 1.1, the exponent of $\eta$ in (1.8) is optimal, as already shown in the symmetric case in $[\mathrm{CD}]$. Also, the conditions $1-(n+\beta) / p+(n+\alpha) / q \geq 0$ or $1-(1+\beta) / p+(1+\alpha) / q>0$ are necessary; see Remark 3.3(iii). Next, for a function $f$ which is only locally Lipschitz continuous, the average $f_{\Omega, \rho^{\alpha}}$ in (1.8) may not be finite. However, as shown at the end of the proof of Theorem 1.1, if $f \in \operatorname{Lip}_{\text {loc }}(\Omega)$ and the right-hand side of $(1.8)$ is finite, then $f_{\Omega, \rho^{\alpha}}$ must be finite. We also note that one can use averages other than $f_{\Omega, \rho^{\alpha}}$ in (1.8); for example, one can use $f_{\mathcal{D}, \rho^{\alpha}}=$ $\int_{\mathcal{D}} f(x) \rho(x)^{\alpha} d x / \rho^{\alpha}(\mathcal{D})$ for any convex domain $\mathcal{D} \subset \Omega$. However, the constant $C$ will then also depend on the ratio $\rho^{\alpha}(\Omega) / \rho^{\alpha}(\mathcal{D})$; see the argument in [CWa, Remark 1.3(1)] and also Remark 3.3(i) below. Finally, we note that the case $q<p$ of Theorem 1.1 is not generally a consequence of the case $q \geq p$ by Hölder's inequality.

\section{Preliminaries}

In what follows, $C$ denotes various positive constants which may differ even in the same string of estimates. We use $C(\alpha, \beta, \cdots)$ to denote a constant which depends only on $\alpha, \beta, \cdots$.

Given a bounded open convex set $\Omega$ and its center $x^{o}$, the class of "balls" naturally associated with $\Omega$ and $x^{o}$ has a very simple geometric structure: it is the collection of all translates and dilates (relative to $x^{o}$ ) of $\Omega$. To be more precise, we begin by defining a quasimetric on $\mathbb{R}^{n}$ which will generate these balls. Let $\Omega-x^{o}$ and $\rho_{o}$ be as in (1.4) and (1.5), and set

$$
\frac{\Omega-x^{o}}{\rho_{o}}=\left\{\frac{x-x^{o}}{\rho_{o}}: x \in \Omega\right\} .
$$

Let $\Phi=\Phi_{x^{o}, \Omega}$ be the gauge or Minkowski function of $\frac{\Omega-x^{o}}{\rho_{o}}$ with respect to the origin:

$\Phi(x)=\Phi_{x^{o}, \Omega}(x)=\inf \left\{t>0: x^{o}+\frac{\rho_{o} x}{t} \in \Omega\right\}=\inf \left\{t>0: \frac{x}{t} \in \frac{\Omega-x^{o}}{\rho_{o}}\right\}, \quad x \in \mathbb{R}^{n}$.

Alternately, $\Phi$ satisfies the formula $\Phi(x)=\rho_{o}|x| / t(x), x \neq 0$, where $t(x)=\sup \{t>$ $\left.0: t x /|x| \in \Omega-x^{o}\right\}$ is the polar description of $\partial\left(\Omega-x^{o}\right)$. 
The main properties of $\Phi$ are

$$
\begin{gathered}
\Phi(x)=0 \quad \text { if and only if } \quad x=0, \\
\Phi(a x)=a \Phi(x), \quad x \in \mathbb{R}^{n}, a \geq 0, \quad \text { and } \\
\Phi(x+y) \leq \Phi(x)+\Phi(y), \quad x, y \in \mathbb{R}^{n} .
\end{gathered}
$$

These properties follow from the definition of $\Phi ;(2.3)$ and (2.4) are standard (see [L, Theorem 3.2]), and (2.2) follows from the fact $\Omega$ is bounded. Furthermore, since $\Omega$ is open, it is evident that (see also [L, Theorem 3.3] and [W, Theorem 5.6.7])

$$
\left\{x \in \mathbb{R}^{n}: \Phi(x)<1\right\}=\frac{\Omega-x^{o}}{\rho_{o}} \text { and }\left\{x \in \mathbb{R}^{n}: \Phi(x)=1\right\}=\partial\left(\frac{\Omega-x^{o}}{\rho_{o}}\right),
$$

or equivalently $\left\{x \in \mathbb{R}^{n}: \Phi(x)<\rho_{o}\right\}=\Omega-x^{o}$ and $\left\{x \in \mathbb{R}^{n}: \Phi(x)=\rho_{o}\right\}=\partial\left(\Omega-x^{o}\right)$.

It follows from (2.1), (1.5) and (1.7) that

$$
\max \{\Phi(x):|x|=1\}=1 \text { and } \min \{\Phi(x):|x|=1\}=1 / \eta .
$$

Hence, by (2.3),

$$
\frac{|x|}{\eta} \leq \Phi(x) \leq|x|, \quad x \in \mathbb{R}^{n} .
$$

By (2.5), (1.5) and (1.7), we also have

$$
1=\min \{|x|: \Phi(x)=1\} \text { and } \eta=\max \{|x|: \Phi(x)=1\} .
$$

Properties (2.2), (2.3) and (2.4) imply that the function $d(x, y)=\Phi(x-y)$ is a quasimetric on $\mathbb{R}^{n}$ with quasimetric constant 1 ; that is, $d(x, y)=0$ if and only if $x=y$ and $d(x, y) \leq d(x, z)+d(z, y)$ for $x, y, z \in \mathbb{R}^{n}$. However, $d(x, y)$ may not equal $d(y, x)$ unless $\Phi$ is symmetric, although, as noted at the beginning of the Appendix, $d(x, y)$ and $d(y, x)$ are equivalent in general by (2.9) below. If $\Phi$ is also symmetric, i.e., if $\Phi(-x)=\Phi(x)$ for all $x$, then the quasimetric $d$ is a metric, $\Phi$ is a norm on $\mathbb{R}^{n}$, and every $\Phi$-ball is symmetric with respect to its center. In general, $\Phi$ is not symmetric, but by using (1.3) we have (see the Appendix)

$$
\Phi(-x) \leq \nu \Phi(x), \quad x \in \mathbb{R}^{n} .
$$

We will denote

$$
B(x, r)=\left\{y \in \mathbb{R}^{n}: \Phi(y-x)<r\right\}, \quad x \in \mathbb{R}^{n}, 0<r<\infty,
$$

and refer to $B(x, r)$ as the $\Phi$-ball with center $x$ and radius $r$. If $B$ is a $\Phi$-ball, we often use $r(B)$ and $x_{B}$ to denote its radius and center. We reserve the notation $c B(x, r)$ for the ball $B(x, c r)$ with radius $c$ times that of $B(x, r)$ and the same center.

Note that by (2.10) and (2.5),

$$
B(0,1)=\left\{x \in \mathbb{R}^{n}: \Phi(x)<1\right\}=\frac{\Omega-x^{o}}{\rho_{o}} \text { and }\left\{x \in \mathbb{R}^{n}: \Phi(x)=1\right\}=\partial(B(0,1)) .
$$

It is now clear that $B(x, r)=x+B(0, r)=x+r B(0,1)$. In particular, every $\Phi$-ball is convex,

$$
\begin{gathered}
B\left(x^{o}, \rho_{o}\right)=\Omega \text { and } \\
|B(x, r)|=\omega_{1} r^{n} \text { where } \omega_{1}=|B(0,1)|=|\Omega| / \rho_{o}^{n} .
\end{gathered}
$$


It will be useful to compare $\eta$ to the Euclidean diameter of the unit $\Phi$-ball $B(0,1)$. Let

$$
\eta_{1}=\sup \left\{\left|x_{1}-y_{1}\right|: x_{1}, y_{1} \in B(0,1)\right\},
$$

be the Euclidean diameter of $B(0,1)$. Then for any $\Phi$-ball $B=B(z, r)$, since $B=$ $z+r B(0,1)$

$$
\operatorname{diam}(B)=\sup \{|x-y|: x, y \in B\}=\eta_{1} r(B) ;
$$

here $\operatorname{diam}(B)$ is the Euclidean diameter of $B$. Then, as we will show in the appendix,

$$
\eta \leq \eta_{1} \leq 2 \eta \text {. }
$$

Any nonnegative function $\phi$ on $\mathbb{R}^{n}$ which satisfies (2.2), (2.3) and (2.4) is called a convex Minkowski function. Its convexity follows easily from (2.3) and (2.4). Conversely, for every convex Minkowski function $\phi$ and every $x^{o} \in \mathbb{R}^{n}$, there is a bounded convex domain $\Omega$ such that

$$
\phi(x)=\inf \left\{t>0: x^{o}+\frac{x}{t} \in \Omega\right\}, \quad x \in \mathbb{R}^{n} .
$$

In fact, $(2.17)$ follows easily if we choose $\Omega$ to be the bounded convex domain $\{x$ : $\left.\phi\left(x-x^{o}\right)<1\right\}$. Hence, for any fixed point $x^{o} \in \mathbb{R}^{n}$, the collection of convex Minkowski functions is the same as the collection of functions for which there is a bounded convex domain $\Omega$ containing $x^{o}$ and satisfying (2.17). Similarly, for any fixed $x^{o} \in \mathbb{R}^{n}$, the collection of bounded convex domains containing $x^{o}$ is the same as the collection of sets $\left\{x: \phi\left(x-x^{o}\right)<1\right\}$ as $\phi$ varies over the class of convex Minkowski functions. We note in passing that the function $\phi(x)=\Phi_{x^{o}, \Omega}(x) / \rho_{o}=\Phi_{x^{o}, \Omega}\left(x / \rho_{o}\right)$ satisfies (2.17).

Any convex Minkowski function $\phi$ can be used to define quasinorm balls which we will call $\phi$-balls, namely

$$
B_{\phi}(x, r)=\left\{z \in \mathbb{R}^{n}: \phi(z-x)<r\right\} .
$$

For a bounded domain $\mathcal{D}$ and a convex Minkowski function $\phi$ (possibly unrelated to $\mathcal{D})$, let

$$
d_{\phi}(x)=d_{\phi, \mathcal{D}}(x)=\min \left\{\phi(y-x): y \in \mathcal{D}^{c}\right\}, \quad x \in \mathcal{D} .
$$

Definition 2.1. Let $\mathcal{D}$ be a bounded domain in $\mathbb{R}^{n}, \phi$ be a convex Minkowski function on $\mathbb{R}^{n}$ and $0<\delta<1 / 2$.

(i) We say a $\phi$-ball $B_{\phi}(x, r)$ is a $\delta$ - $\phi$-ball (in $\mathcal{D}$ ) if $x \in \mathcal{D}$ and $r \leq \delta d_{\phi}(x)$. When equality holds, i.e., when $r=\delta d_{\phi}(x)$, we say the ball is a $\delta$-Whitney $\phi$-ball.

(ii) We say a measure $\sigma$ is $\phi$-doubling on $\mathcal{D}$ if there exists $D_{\sigma}>0$ such that

$$
\sigma\left(B_{\phi}\left(x, 2^{k} r\right)\right) \leq D_{\sigma}^{k} \sigma\left(B_{\phi}(x, r)\right), k \in \mathbb{N},
$$

for all $x \in \mathcal{D}$ and $r \leq \operatorname{diam}(\mathcal{D})$. If (2.19) holds for all $\phi$-balls $B_{\phi}(x, r)$ in $\mathbb{R}^{n}$, we say $\sigma$ is $\phi$-doubling. If (2.19) holds only when $B_{\phi}(x, r)$ is a $\delta$ - $\phi$-ball, we say $\sigma$ is $\delta$ - $\phi$-doubling. Some discussion regarding $\delta$ - $\phi$-doubling can be found in $[\mathrm{CWa}]$ and $[\mathrm{CWb}]$ when $\phi$ is symmetric.

We now consider domains which satisfy the Boman chain condition for $\phi$-balls. As usual, we denote $c B=B_{\phi}(x, c r)$ if $B=B_{\phi}(x, r)$ and $c>0$. 
Definition 2.2. Let $0<\delta<1 / 2, M_{1}, M_{2}>1$, and $\phi$ be a convex Minkowski function on $\mathbb{R}^{n}$. For a bounded domain $\mathcal{D} \in \mathbb{R}^{n}$, we write $\mathcal{D} \in \mathcal{F}\left(\delta, M_{1}, M_{2}\right)=\mathcal{F}_{\phi}\left(\delta, M_{1}, M_{2}\right)$ if $\mathcal{D}$ has a Boman cover $W$ consisting of $\delta$-Whitney $\phi$-balls, that is, if there exists a countable collection $W=\{B\}$ of $\delta$-Whitney $\phi$-balls such that

(i) $\mathcal{X}_{\mathcal{D}} \leq \sum_{B \in W} \mathcal{X}_{B} \leq M_{1} \mathcal{X}_{\mathcal{D}}$

(ii) for any $B \in W$, there is a finite sequence $\left\{B=B_{0}, B_{1}, \ldots, B_{K}\right\}$ of balls in $W$, where $K$ may depend on $B$ but $B_{K}=B^{*}$ is independent of $B$, such that $B \subset M_{2} B_{j}$ for $j=1, \ldots, K$, and for each $i<K$, there exists a $\phi$-ball $B_{i}^{\prime} \subset B_{i+1} \cap B_{i}$ with $B_{i} \cup B_{i+1} \subset M_{1} B_{i}^{\prime}$.

If $\mathcal{D} \in \mathcal{F}_{\phi}\left(\delta, M_{1}, M_{2}\right)$ for some $M_{1}, M_{2}$ and $\delta, 0<\delta<1 / 2$, we say $\mathcal{D}$ is a $\phi$-Boman domain and call $B^{*}$ the central $\phi$-ball of $\mathcal{D}$. See also $[\mathrm{C} 09, \mathrm{CWa}$. Note that the notions of $\delta$ - $\phi$-doubling and $\phi$-doubling are the same on Boman domains; in the case of a symmetric quasimetric, see [CWa, Proposition 2.2(2)].

Before we proceed to show that a $\Phi$-ball is a $\Phi$-Boman domain, $\Phi=\Phi_{x^{o}, \Omega}$, let us list some simple facts. As usual, we denote $\Phi$-balls $B_{\Phi}(x, r)$ simply by $B(x, r)$.

Lemma 2.3. Let $\Omega$ be a bounded open convex set with center $x^{\circ}=0$ and $\Phi=\Phi_{0, \Omega}$ be as in (2.1), so that $\Omega=B\left(0, \rho_{o}\right)$ with $\rho_{o}=\operatorname{dist}(0, \partial \Omega)=r(\Omega)$. Let $z^{\prime}$ be a unit vector, and denote $\Phi\left(z^{\prime}\right)=\xi$ and $\Phi\left(-z^{\prime}\right)=\bar{\xi}$.

(i) If $0<t<\rho_{o} / \xi$, then $t z^{\prime} \in \Omega$ and $d_{\Phi}\left(t z^{\prime}\right)=\rho_{o}-t \xi$. Equivalently, if $z \in \Omega$, then $d_{\Phi}(z)=\rho_{o}-\Phi(z)$.

(ii) If $0<t_{1}<t_{0}$, then $B\left(t_{1} z^{\prime}, r_{1} \bar{\xi}\right) \subset B\left(t_{0} z^{\prime}, r_{0} \bar{\xi}\right)$ if and only if $t_{0}-t_{1}+r_{1} \leq r_{0}$.

(iii) If $0<\delta<1 / 2,0<t<t_{0}<\rho_{o} / \xi$ and $B\left(t_{1} z^{\prime}, r\right)$ is a $\delta$-Whitney $\Phi$-ball in $\Omega$ which is not a subset of $B\left(t_{0} z^{\prime}, r_{0}\right)$, then $r \geq C(\delta, \nu) r_{0}$.

(iv) If $x, y \in \Omega, 0<\delta<1 / 2$ and $B\left(x, \delta d_{\Phi}(x)\right)$ intersects $B\left(y, \delta d_{\Phi}(y)\right)$, then $d_{\Phi}(y) \leq(2+\nu) d_{\Phi}(x)$ and $B\left(y, \delta d_{\Phi}(y)\right) \subset[(1+\nu)(2+\nu)+1] B\left(x, \delta d_{\Phi}(x)\right)$. If $B_{1}, B_{2}$ are any two $\Phi$-balls whose closures intersect and $r\left(B_{1}\right) \leq \alpha r\left(B_{2}\right)$, then $B_{1} \subset[\alpha(1+\nu)+1] B_{2}$.

As we have been unable to locate a reference for these facts, we prove them in the Appendix.

Proposition 2.4. Let $\Omega$ be a bounded convex domain in $\mathbb{R}^{n}$, and let $\Phi=\Phi_{x^{o}, \Omega}$ be defined by (2.1) and $d_{\Phi}$ by (2.18). (Hence $\Omega$ is the $\Phi$-ball $B\left(x^{o}, \rho_{o}\right)$ and $\rho_{o}=d_{\Phi}\left(x^{o}\right)$.) If $0<\delta<1 / 2$, then $\Omega \in \mathcal{F}\left(\delta, M_{1}, M_{2}\right)=\mathcal{F}_{\Phi}\left(\delta, M_{1}, M_{2}\right)$ where $M_{1}, M_{2}$ depend only on $n$ and $\delta$, and the central ball is $B\left(x^{o}, \delta \rho_{o}\right)$. Moreover, for any $x_{0} \in \Omega, x_{0} \neq x^{o}$, there exists a chain of $\delta$-Whitney $\Phi$-balls $\left\{Q_{0}=B\left(x_{0}, \delta d_{\Phi}\left(x_{0}\right)\right), Q_{1}, \cdots, Q_{N}=B\left(x^{o}, \delta \rho_{o}\right)\right\}$ $\left(N\right.$ may depend on $\left.x_{0}\right)$, with bounded intercepts depending only on $n$, such that for each $i, Q_{0} \subset M_{2} Q_{i}$ and there exists a $\Phi$-ball $\tilde{Q}_{i} \subset Q_{i-1} \cap Q_{i}$ with $Q_{i-1} \cup Q_{i} \subset M_{1} \tilde{Q}_{i}$.

This can be proved by methods like those in [CD], with the help of Lemma 2.3; see the Appendix.

We also need the next two variants of known results.

Theorem 2.5. Let $\phi$ be a convex Minkowski function in $\mathbb{R}^{n}$ and $\Omega \in \mathcal{F}_{\phi}\left(\delta, M_{1}, M_{2}\right)$ for some $\delta, M_{1}, M_{2}, 0<\delta<1 / 2$, with Boman cover $W$ and central $\phi$-ball $B^{*}$. Let $f$ be a Borel measurable function on $\Omega$ and $w$ be a $\delta$ - $\Phi$-doubling Borel measure. For 
each $\phi$-ball $B \in W$, let $a(f, B)$ be an arbitrary constant associated with $B$. Then if $1 \leq q<\infty$,

$$
\left\|f-a\left(f, B^{*}\right)\right\|_{L_{w}^{q}(\Omega)}^{q} \leq C\left(q, D_{w}, M_{1}, M_{2}\right) \sum_{B \in W}\|f-a(f, B)\|_{L_{w}^{q}(B)}^{q} .
$$

Theorem 2.5 was first obtained in [C93, Theorem 1.5] for the usual Euclidean metric and a doubling weight $w$. That proof can be easily extended to the present case using the modified maximal function

$$
M_{w}^{\delta} f(x)=\sup \left\{\frac{1}{w(B)} \int_{B}|f| d w: \quad B \text { is a } \delta \text { - } \phi \text {-ball containing } x\right\} .
$$

More details can be found in [CWa], and a further extension is given in [CW6].

Proposition 2.6. Let $\Omega$ be a bounded convex domain in $\mathbb{R}^{n}$ and $\Phi=\Phi_{x^{o}, \Omega}$. If $\mu$ is $\Phi$-doubling on $\Omega$, then there exist $A_{1}, A_{2}>0$ depending only on $D_{\mu}$ such that for all $x \in \Omega$ and $0<r<\rho_{o}$,

$$
A_{1} \leq \frac{\mu(B(x, 2 r))}{\mu(B(x, r))} \leq A_{2} .
$$

The proof is a simple adaptation of the argument of [Wh, p.269]; see also [C09, Proposition 2.8] and [CWa, Proposition 2.3].

We now derive a weak-type self-improving property of weighted Poincaré inequalities on convex domains.

Proposition 2.7. Suppose $\Omega$ is a bounded convex domain in $\mathbb{R}^{n}$ and $\Phi=\Phi_{x^{o}, \Omega}$. Let $\mu, \sigma$ be $\Phi$-doubling Borel measures on $\Omega, 0<\delta<1 / 2$, and $a(B)$ be a nonnegative set function defined for all $\delta$ - $\Phi$-balls $B$. If $f$ is a Borel function on $\Omega$ such that for all $\delta$ - $\Phi$-balls $B$,

$$
\frac{1}{\sigma(B)}\left\|f-f_{B, \sigma}\right\|_{L_{\sigma}^{1}(B)} \leq a(B)
$$

and if there exist constants $q, \theta, C_{\Omega}$ with $1 \leq q<\infty, 0<\theta<1$ and $C_{\Omega}>0$ such that

$$
\sum_{B \in I}\left[a(B)^{q} \mu(B)\right]^{\theta} \leq C_{\Omega}^{\theta}
$$

for every collection I of disjoint $\delta$ - $\Phi$-balls $B$, then

$$
\sup _{t>0} t \mu\left\{x \in \Omega:\left|f(x)-f_{\Omega, \mu}\right|>t\right\}^{\frac{1}{q}} \leq C C_{\Omega}^{\frac{1}{q}},
$$

where $C=C\left(D_{\sigma}, D_{\mu}, \delta, n, \theta, q\right)$ and $C_{\Omega}$ is the constant in (2.22).

Proof. The result will be a corollary of [CWa, Theorem 1.2] concerning abstract measure spaces. We denote $\Phi$-balls simply by $B(x, r)$. As usual, $\Omega=B\left(x^{o}, \rho_{o}\right)$, and we may assume without loss of generality that $x^{o}=0$. For any $x \neq 0$, from Proposition 2.4 , there exists a finite sequence of $\delta$-Whitney $\Phi$-balls $\left\{Q_{0}^{x}, Q_{1}^{x}, \cdots, Q_{N}^{x}\right\}, N=N(x)$, with bounded intercepts (depending only on $n$ ) and centers along the line segment $\overline{x 0}$, such that $Q_{0}^{x}=B\left(0, \delta \rho_{o}\right), Q_{N}^{x}=B\left(x, \delta d_{\Phi}(x)\right)$, and

$$
\sigma\left(Q_{j}^{x} \cup Q_{j+1}^{x}\right) \leq C \sigma\left(Q_{j}^{x} \cap Q_{j+1}^{x}\right), \quad j=0, \ldots, N-1,
$$

since $\sigma$ is $\Phi$-doubling. Choose the least $k=k(x) \in \mathbb{N}$ such that $\cup_{j=0}^{N} Q_{j}^{x} \subset 2^{k} Q_{N}^{x}$. For $i=0,1, \ldots$, define $B_{i}^{x}=2^{k-i} Q_{N}^{x}$, and for $j>N$, define $Q_{j}^{x}=B_{j-N+k}^{x}$. Note that 
$Q_{j}^{x}$ has center $x$ if $j \geq N$, and that $Q_{j}^{x}$ is a $\delta$-Whitney ball if $j \leq N$. If $x=0$ and $i=1,2, \ldots$, define $Q_{i}^{x}, B_{i}^{x}$ by $Q_{i}^{x}=B_{i}^{x}=2^{-i} B\left(0, \delta \rho_{o}\right)$. For $x \in \Omega$ and $i \geq 0$, let

$$
\mathcal{C}\left(B_{i}^{x}\right)=\left\{Q_{j}^{x}: Q_{j}^{x} \subset B_{i}^{x}, Q_{j}^{x} \not \subset \frac{1}{2} B_{i}^{x}\right\} .
$$

It follows that if $Q_{j}^{x} \in \mathcal{C}\left(B_{i}^{x}\right)$, then $r\left(Q_{j}^{x}\right) \leq r\left(B_{i}^{x}\right) \leq C(\delta, \nu) r\left(Q_{j}^{x}\right)$; in fact, for those $Q_{j}^{x}$ which are $\delta$-Whitney balls, this is true by Lemma 2.3(iii), and for the rest, it follows from their definition. Hence $B_{i}^{x} \subset C(\delta, \nu) Q_{j}^{x}$ if $Q_{j}^{x} \in \mathcal{C}\left(B_{i}^{x}\right)$. Also, there is a constant $C(\delta, n)$ so that for any fixed $x$ and $i$, the number of $Q_{j}^{x}$ in $\mathcal{C}\left(B_{i}^{x}\right)$ is bounded by $C(\delta, n)$; this follows from a volume argument since those $Q_{j}^{x}$ with $j \leq N$ have bounded intercepts depending only on $n$ and $\delta$.

Next, if $\left\{B_{\alpha}\right\} \subset\left\{B_{i}^{x}: x \in \Omega, i \geq 0\right\}$ is a family of pairwise disjoint balls, then since the number of $Q$ in each $\mathcal{C}\left(B_{\alpha}\right)$ is bounded by $L=C(\delta, n)$, we have

$$
\begin{aligned}
& \sum_{\alpha}\left[\left(\sum_{Q \in \mathcal{C}\left(B_{\alpha}\right)} a(Q)\right)^{q} \mu\left(B_{\alpha}\right)\right]^{\theta} \\
\leq & \sum_{\alpha}\left[L^{q / q^{\prime}} \sum_{Q \in \mathcal{C}\left(B_{\alpha}\right)} a(Q)^{q} \mu\left(B_{\alpha}\right)\right]^{\theta} \\
\leq & {\left[C\left(D_{\mu}, \delta, \nu\right) L^{q / q^{\prime}}\right]^{\theta} \sum_{\alpha} \sum_{Q \in \mathcal{C}\left(B_{\alpha}\right)}\left[a(Q)^{q} \mu(Q)\right]^{\theta}, }
\end{aligned}
$$

where to obtain the last inequality we used $0<\theta<1$ and $\mu\left(B_{\alpha}\right) \leq C\left(D_{\mu}, \delta, \nu\right) \mu(Q)$ for $Q \in \mathcal{C}\left(B_{\alpha}\right)$. Since the balls $Q$ in $\mathcal{C}\left(B_{\alpha}\right)$ have uniformly bounded intercepts, there exists $K=K(\delta, n)$ such that each $\mathcal{C}\left(B_{\alpha}\right)$ can be decomposed into the union of at most $K$ families of pairwise disjoint balls. Since the $B_{\alpha}$ are also pairwise disjoint, (2.22) implies that

$$
\sum_{\alpha}\left[\left(\sum_{Q \in \mathcal{C}\left(B_{\alpha}\right)} a(Q)\right)^{q} \mu\left(B_{\alpha}\right)\right]^{\theta} \leq K\left[C\left(D_{\mu}, \delta, \nu\right) L^{q / q^{\prime}}\right]^{\theta} C_{\Omega}^{\theta} .
$$

Finally, a Vitali type covering argument like that in the proof of Proposition 2.4 shows that any family of $\Phi$-balls $\left\{B\left(x, r_{x}\right), x \in E\right\}$ has a subfamily $\mathcal{F}$ of pairwise disjoint $\Phi$-balls such that

$$
\mu(E) \leq C\left(D_{\mu}, \nu\right) \sum_{\mathcal{F}} \mu\left(B\left(x_{i}, r_{i}\right)\right)
$$

Proposition 2.7 now follows by applying [CWa, Theorem 1.2].

\section{Proof of the main theorem}

First we derive an unweighted Poincaré inequality on convex domains similar to (1.1) when $1 / q \geq 1 / p-1 / n$. The point of the estimate is that it improves the well-known inequality $(\mathrm{P})$ by showing that the norm constant is independent of the domain's eccentricity. 
Proposition 3.1. Let $\Omega$ be a bounded convex domain in $\mathbb{R}^{n}$. If $1 \leq p, q<\infty$ and $1+\frac{n}{q}-\frac{n}{p} \geq 0$, then for all $f \in \operatorname{Lip}_{\text {loc }}(\Omega)$,

$$
\left\|f-f_{\Omega}\right\|_{L^{q}(\Omega)} \leq C(p, q, n)|\Omega|^{\frac{1}{q}-\frac{1}{p}} \operatorname{diam}(\Omega)\|\nabla f\|_{L^{p}(\Omega)} \quad \text { with } f_{\Omega}=\frac{1}{|\Omega|} \int_{\Omega} f d x .
$$

Proof. By Hölder's inequality, we only need to consider the case $q>p$. In fact, the case $q \leq p$ has already been given in (1.1) with $\alpha=0$. Fix $\Omega$ and let $\Phi=\Phi_{x^{o}, \Omega}$. If $f \in \operatorname{Lip}_{l o c}(\Omega)$ and $B$ is any $\Phi$-ball in $\Omega$, then since $B$ is convex, we know by (1.1) with $\alpha=0$ and $q=1$ that

$$
\frac{1}{|B|}\left\|f-f_{B}\right\|_{L^{1}(B)} \leq C(p) \frac{\operatorname{diam}(B)}{|B|^{1 / p}}\|\nabla f\|_{L^{p}(B)} .
$$

Set

$$
a(B)=\frac{\operatorname{diam}(B)}{|B|^{1 / p}}\|\nabla f\|_{L^{p}(B)} .
$$

Let $\omega_{1}=|B(0,1)|$ be the volume of the unit $\Phi$-ball, and recall from (2.15) and (2.13) that $\operatorname{diam}(B)=\eta_{1} r(B)$ and $|B|=\omega_{1} r(B)^{n}$ if $B$ is any $\Phi$-ball. Let us now fix $0<\delta<1 / 2$. If $I$ is a collection of disjoint $\delta$ - $\Phi$-balls in $\Omega$, then

$$
\begin{aligned}
\sum_{B_{\alpha} \in I} a\left(B_{\alpha}\right)^{p}\left|B_{\alpha}\right|^{\frac{p}{q}} & =\sum_{B_{\alpha} \in I} \eta_{1}^{p} \omega_{1}^{p\left(\frac{1}{q}-\frac{1}{p}\right)} r\left(B_{\alpha}\right)^{\left(1+\frac{n}{q}-\frac{n}{p}\right) p}\|\nabla f\|_{L^{p}\left(B_{\alpha}\right)}^{p} \\
& \leq \eta_{1}^{p} \omega_{1}^{p\left(\frac{1}{q}-\frac{1}{p}\right)} r(\Omega)^{\left(1+\frac{n}{q}-\frac{n}{p}\right) p} \sum_{B_{\alpha} \in I}\|\nabla f\|_{L^{p}\left(B_{\alpha}\right)}^{p}
\end{aligned}
$$

since $1+\frac{n}{q}-\frac{n}{p} \geq 0$. It is now clear that

$$
\sum_{B_{\alpha} \in I} a\left(B_{\alpha}\right)^{p}\left|B_{\alpha}\right|^{\frac{p}{q}} \leq\left(|\Omega|^{\frac{1}{q}-\frac{1}{p}} \operatorname{diam}(\Omega)\|\nabla f\|_{L^{p}(\Omega)}\right)^{p} .
$$

Hence (2.22) holds with $\theta=p / q$ and $C_{\Omega}=\left(|\Omega|^{\frac{1}{q}-\frac{1}{p}} \operatorname{diam}(\Omega)\|\nabla f\|_{L^{p}(\Omega)}\right)^{q}$. It then follows from Proposition 2.7 that

$$
\sup _{t>0} t\left|\left\{x \in \Omega:\left|f(x)-f_{\Omega}\right|>t\right\}\right|^{\frac{1}{q}} \leq C(p, q, n)|\Omega|^{\frac{1}{q}-\frac{1}{p}} \operatorname{diam}(\Omega)\|\nabla f\|_{L^{p}(\Omega)},
$$

where $f_{\Omega}=\int_{\Omega} f d x /|\Omega|$. This proves the weak-type version of (3.1). The strong-type estimate (3.1) itself now follows by a well-known truncation technique. We omit the details but refer to the proof of [CWa, Theorem 1.10] for the argument.

Next, we estimate the number of disjoint Whitney balls of a given size.

Lemma 3.2. Let $\Omega$ be a bounded open convex domain in $\mathbb{R}^{n}$, and $\Phi=\Phi_{x^{o}, \Omega}$. If $0<\delta<1 / 2$, then for each $k \in \mathbb{N}$, the number of pairwise disjoint $\delta$-Whitney $\Phi$-balls with radius between $2^{-k-1} r(\Omega)$ and $2^{-k} r(\Omega)$ is at most $C(\delta, n) 2^{(n-1) k}$.

Proof. We may assume that $x^{o}=0$. As usual, let $\rho_{o}=r(\Omega)$. If $B=B\left(z, \delta d_{\Phi}(z)\right)$ is a $\delta$-Whitney $\Phi$-ball with $2^{-k-1} \rho_{o} \leq r(B) \leq 2^{-k} \rho_{o}$, then for any $x \in B$,

$d_{\Phi}(x) \leq d_{\Phi}(z)+\Phi(z-x) \leq d_{\Phi}(z)+\nu \delta d_{\Phi}(z)=\delta d_{\Phi}(z)\left(\delta^{-1}+\nu\right) \leq 2^{-k} \rho_{o}\left(\delta^{-1}+\nu\right)$.

Since $d_{\Phi}(x)=\rho_{o}-\Phi(x)$ by Lemma 2.3(i), we obtain $\rho_{o}\left(1-2^{-k}\left(\delta^{-1}+\nu\right)\right) \leq \Phi(x)<\rho_{o}$, and therefore

$$
B \subset B\left(0, \rho_{o}\right) \backslash B\left(0, \rho_{o}\left(1-2^{-k}\left(\delta^{-1}+\nu\right)\right), \text { assuming that } 2^{-k}\left(\delta^{-1}+\nu\right)<1 .\right.
$$


This annular region has volume

$$
\omega_{1} \rho_{o}^{n}\left[1-\left\{1-2^{-k}\left(\delta^{-1}+\nu\right)\right\}^{n}\right] \leq|\Omega| n 2^{-k}\left(\delta^{-1}+\nu\right) \leq C(\delta, n) 2^{-k}|\Omega| .
$$

By volume considerations, it follows that the number of pairwise disjoint $\delta$-Whitney $\Phi$-balls with radius between $2^{-k-1} \rho_{o}$ and $2^{-k} \rho_{o}$ is at most

$$
\frac{C(\delta, n) 2^{-k}|\Omega|}{\omega_{1}\left(2^{-k-1} \rho_{o}\right)^{n}}=C(\delta, n) 2^{k(n-1)},
$$

which completes the proof in case $2^{-k}\left(\delta^{-1}+\nu\right)<1$. The argument for the remaining values of $k$ is similar provided the role of the annular region is played by all of $\Omega$.

Proof of Theorem 1.1. Let $\Omega$ be a bounded convex domain, $\Phi=\Phi_{x^{\circ}, \Omega}$, and recall that $\rho(x)=\min _{y \in \Omega^{c}}|x-y|$. Then $\rho=0$ on $\Omega^{c}$ and $\rho$ is a concave function on $\Omega$; for the latter, see for example [CW3, Remark 1.5(4)]. If $B$ is a $\Phi$-ball in $\Omega$, we set

$$
\bar{\rho}(B)=\sup \{\rho(x): x \in B\} .
$$

By $(2.15)$ and (1.6), $\operatorname{diam}(B)=\eta_{1} r(B)$ and $r(\Omega)=\rho_{o} \leq \bar{\rho}(\Omega) \leq(1+\nu) \rho_{o}$. Also, for any $\Phi$-ball $B \subset \Omega$, we have $r(B) \leq \bar{\rho}(B)$ since

$$
\bar{\rho}(B) \geq \rho\left(x_{B}\right) \geq \sup _{y \in \partial B}\left|y-x_{B}\right| \geq \sup _{y \in \partial B} \Phi\left(y-x_{B}\right)=r(B),
$$

where we used (2.7) to obtain the last inequality.

We claim that there exists $0<\tau<1$ depending only on $n$ (in fact, depending only on $\nu$ ) such that if $B$ is a $\Phi$-ball with $2 B \subset \Omega$, then for all $x \in B$,

$$
\tau \rho\left(x_{B}\right)<\rho(x)<\tau^{-1} \rho\left(x_{B}\right)
$$

and hence

$$
\tau^{2} \bar{\rho}(B) \leq \rho(x) \leq \bar{\rho}(B) \text { if } x \in B \text { and } 2 B \subset \Omega
$$

Let us first show that if $B$ is a $\Phi$-ball, $x \in B$ and $0 \leq k \leq 2 / \nu$, then $2 x-x_{B}, k\left(x_{B}-\right.$ $x)+x_{B} \in 2 B$. For such $B, x$ and $k$,

$$
\begin{gathered}
\Phi\left(\left[2 x-x_{B}\right]-x_{B}\right)=2 \Phi\left(x-x_{B}\right)<2 r(B) \quad \text { and } \\
\Phi\left(\left[k\left(x_{B}-x\right)+x_{B}\right]-x_{B}\right)=k \Phi\left(x_{B}-x\right) \leq k \nu \Phi\left(x-x_{B}\right)<k \nu r(B)<2 r(B),
\end{gathered}
$$

as desired. In particular, if in addition $2 B \subset \Omega$, then both $2 x-x_{B}, k\left(x_{B}-x\right)+x_{B} \in \Omega$. Since $x$ is the midpoint of the line segment connecting $x_{B}$ and $2 x-x_{B}$, it follows from concavity of $\rho$ that

$$
\rho(x) \geq \frac{1}{2} \rho\left(x_{B}\right)+\frac{1}{2} \rho\left(2 x-x_{B}\right)>\frac{1}{2} \rho\left(x_{B}\right) .
$$

Similarly, choosing $k=2 / \nu$ and $\theta=k /(1+k)$, we have $k\left(x_{B}-x\right)+x_{B} \in \Omega$ and $x_{B}=\theta x+(1-\theta)\left\{k\left(x_{B}-x\right)+x_{B}\right\}$, and consequently

$$
\rho\left(x_{B}\right) \geq \theta \rho(x)+(1-\theta) \rho\left(k\left(x-x_{B}\right)+x_{B}\right)>\theta \rho(x),
$$

which proves the claim. 
Hence, assuming that $2 B \subset \Omega$ and applying (3.1) with $\Omega$ replaced by $B$, if $1 \leq$ $p \leq q<\infty, 1+\frac{n}{q}-\frac{n}{p} \geq 0$ and $\alpha, \beta \in \mathbb{R}$, we have (with $f_{B}=|B|^{-1} \int_{B} f d x$ and $C=C(p, q, \alpha, \beta, n))$

$$
\begin{aligned}
\left\|f-f_{B}\right\|_{L_{\rho^{\alpha}}^{q}(B)} & \leq C \bar{\rho}(B)^{\frac{\alpha}{q}-\frac{\beta}{p}}|B|^{\frac{1}{q}-\frac{1}{p}} \operatorname{diam}(B)\|\nabla f\|_{L_{\rho^{\beta}}^{p}(B)} \\
& =C \eta_{1} \omega_{1}^{\frac{1}{q}-\frac{1}{p}} \bar{\rho}(B)^{\frac{\alpha}{q}-\frac{\beta}{p}} r(B)^{1+\frac{n}{q}-\frac{n}{p}}\|\nabla f\|_{L_{\rho^{\beta}}^{p}(B)} .
\end{aligned}
$$

We now fix $0<\delta<1 / 2$. If $W$ is a Boman cover of $\Omega$ as in Proposition 2.4, then every $B \in W$ is a $\delta$-Whitney $\Phi$-ball with $2 B \subset \Omega$. Therefore, if $B \in W$, since $\bar{\rho}(B) \geq r(B)$ and $1+\frac{n}{q}-\frac{n}{p} \geq 0$, it follows from the previous estimate that

$$
\begin{aligned}
\left\|f-f_{B}\right\|_{L_{\rho^{\alpha}}^{q}(B)} & \leq C \eta_{1} \omega_{1}^{\frac{1}{q}-\frac{1}{p}} \bar{\rho}(B)^{1+\frac{n+\alpha}{q}-\frac{n+\beta}{p}}\|\nabla f\|_{L_{\rho^{\beta}}^{p}(B)} \\
& \leq C \eta_{1} \omega_{1}^{\frac{1}{q}-\frac{1}{p}} \rho_{o}^{1+\frac{n+\alpha}{q}-\frac{n+\beta}{p}}\|\nabla f\|_{L_{\rho^{\beta}}^{p}(B)}
\end{aligned}
$$

since $\bar{\rho}(B) \leq \bar{\rho}(\Omega) \sim \rho_{o}$ and by hypothesis $1+\frac{n+\alpha}{q}-\frac{n+\beta}{p} \geq 0$.

Next, we will show that if $\alpha \geq 0$ then $\rho^{\alpha}$ is $\Phi$-doubling on $\Omega$ (see Definition 2.1(ii)) with doubling constant depending only on $n$ and $\alpha$. It is enough to show that

$$
\int_{2 B} \rho^{\alpha} d x \leq 2^{n+\alpha} \int_{B} \rho^{\alpha} d x \quad \text { for any } \Phi \text {-ball } B \text { with center in } \Omega \text {. }
$$

First note that $\rho(x) \leq 2 \rho\left(\frac{x_{B}+x}{2}\right)$ if $x \in \mathbb{R}^{n}$. This is obvious if $x \notin \Omega$ since then $\rho(x)=0$, while if $x \in \Omega$ (recall $x_{B} \in \Omega$ too),

$$
\rho\left(\frac{x_{B}+x}{2}\right) \geq \frac{1}{2} \rho\left(x_{B}\right)+\frac{1}{2} \rho(x)>\frac{1}{2} \rho(x)
$$

by concavity of $\rho$. Therefore, since $\alpha \geq 0$,

$$
\begin{aligned}
& \int_{2 B} \rho(x)^{\alpha} d x \leq 2^{\alpha} \int_{\Phi\left(x-x_{B}\right)<2 r(B)} \rho\left(\frac{x_{B}+x}{2}\right)^{\alpha} d x \\
& =2^{\alpha+n} \int_{\Phi\left(y-x_{B}\right)<r(B)} \rho(y)^{\alpha} d y=2^{\alpha+n} \int_{B} \rho(y)^{\alpha} d y
\end{aligned}
$$

as desired.

Recall that $\Omega \in \mathcal{F}_{\Phi}\left(\delta, M_{1}, M_{2}\right)$, with $M_{1}, M_{2}$ depending only on $\delta, n$. Hence, by Theorem 2.5, if $B^{*}$ is the central ball in $\Omega$, we have for $\alpha \geq 0$ and $1 \leq q<\infty$ that

$$
\left\|f-f_{B^{*}}\right\|_{L_{\rho^{\alpha}}^{q}(\Omega)}^{q} \leq C(q, \alpha, n) \sum_{B \in W}\left\|f-f_{B}\right\|_{L_{\rho^{\alpha}}^{q}(B)}^{q} .
$$

In order to estimate the sum in (3.7) in case $q \geq p$ and $1+(n+\alpha) / q-(n+\beta) / p \geq 0$, we will use (3.6). Since $q \geq p$ and the balls $B \in W$ have bounded overlaps (see Definition 2.1(i)), we have

$$
\sum_{B \in W}\|\nabla f\|_{L_{\rho^{\beta}}^{p}(B)}^{q}=\sum_{B \in W}\left(\int_{B}|\nabla f|^{p} \rho^{\beta} d x\right)^{\frac{q}{p}}
$$




$$
\leq\left(\sum_{B \in W} \int_{B}|\nabla f|^{p} \rho^{\beta} d x\right)^{\frac{q}{p}} \leq C(n, p, q)\|\nabla f\|_{L_{\rho^{\beta}}^{p}(\Omega)}^{q},
$$

and then

$$
\begin{gathered}
\left\|f-f_{B^{*}}\right\|_{L_{\rho^{\alpha}}^{q}(\Omega)} \leq C(p, q, \alpha, \beta, n) \eta_{1} \omega_{1}^{\frac{1}{q}-\frac{1}{p}} \rho_{o}^{1+\frac{\alpha+n}{q}-\frac{\beta+n}{p}}\|\nabla f\|_{L_{\rho^{\beta}}^{p}(\Omega)} \\
=C(p, q, \alpha, \beta, n) \eta_{1}^{\frac{\beta}{p}-\frac{\alpha}{q}} \operatorname{diam}(\Omega)^{1+\frac{\alpha}{q}-\frac{\beta}{p}}|\Omega|^{\frac{1}{q}-\frac{1}{p}}\|\nabla f\|_{L_{\rho^{\beta}}^{p}(\Omega)}
\end{gathered}
$$

as $|\Omega|=\omega_{1} \rho_{o}^{n}$ and $\operatorname{diam}(\Omega)=\eta_{1} \rho_{o}$.

If $1 \leq q<p<\infty$, we will only consider the case $\alpha / q-\beta / p \leq 0$ as the other case is easier. As noted at the beginning of the proof, $r(B) \leq \bar{\rho}(B)$ for any $\Phi$-ball $B \subset \Omega$, and consequently $\bar{\rho}(B)^{\alpha / q-\beta / p} \leq r(B)^{\alpha / q-\beta / p}$. By (3.7), (3.5) and Hölder's inequality with exponents $p / q, p /(p-q)$,

$$
\begin{aligned}
& \left\|f-f_{B^{*}}\right\|_{L_{\rho^{\alpha}}^{q}(\Omega)}^{q} \leq \\
& \left(C(p, q, \alpha, \beta, n) \eta_{1} \omega_{1}^{\frac{1}{q}-\frac{1}{p}}\right)^{q}\left(\sum_{B \in W} r(B)^{\left(1+\frac{n+\alpha}{q}-\frac{n+\beta}{p}\right) \frac{q p}{p-q}}\right)^{\frac{p-q}{p}}\|\nabla f\|_{L_{\rho^{\beta}}^{p}(\Omega)}^{q} .
\end{aligned}
$$

But

$$
\left(\sum_{B \in W} r(B)^{\left(1+\frac{n+\alpha}{q}-\frac{n+\beta}{p}\right) \frac{q p}{p-q}}\right)^{\frac{p-q}{p}} \leq\left(\sum_{k \in \mathbb{N}} \sum_{\substack{B \in W \\ r(B) \sim 2^{-k} \rho_{o}}} r(B)^{\left(1+\frac{n+\alpha}{q}-\frac{n+\beta}{p}\right) \frac{q p}{p-q}}\right)^{\frac{p-q}{p}},
$$

which by Lemma 3.2 and the hypothesis $1+\frac{\alpha}{q}-\frac{\beta}{p}>-\frac{p-q}{p q}$ is at most

$$
\left(\sum_{k \in \mathbb{N}} C(n) 2^{(n-1) k}\left(2^{-k} \rho_{o}\right)^{\left(1+\frac{n+\alpha}{q}-\frac{n+\beta}{p}\right) \frac{q p}{p-q}}\right)^{\frac{p-q}{p}} \leq C(n, p, q, \beta, \alpha) \rho_{o}^{q\left(1+\frac{n+\alpha}{q}-\frac{n+\beta}{p}\right)} .
$$

Hence, we again obtain that

$$
\left\|f-f_{B^{*}}\right\|_{L_{\rho^{\alpha}}^{q}(\Omega)} \leq C(p, q, \beta, \alpha, n) \eta_{1}^{\frac{\beta}{p}-\frac{\alpha}{q}} \operatorname{diam}(\Omega)^{1+\frac{\alpha}{q}-\frac{\beta}{p}}|\Omega|^{\frac{1}{q}-\frac{1}{p}}\|\nabla f\|_{L_{\rho^{\beta}}^{p}(\Omega)} .
$$

Finally, assuming that the right sides of (3.8) and (3.9) are finite, i.e., that $|\nabla f| \in$ $L_{\rho^{\beta}}^{p}(\Omega)$, and noting that $f_{B^{*}}$ is finite since $f \in \operatorname{Lip}_{l o c}(\Omega)$, it follows that $f \in L_{\rho^{\alpha}}^{q}(\Omega)$. Hence $f_{\Omega, \rho^{a}}$ is finite, and we easily obtain

$$
\left\|f-f_{\Omega, \rho^{\alpha}}\right\|_{L_{\rho^{\alpha}}^{q}(\Omega)} \leq 2\left\|f-f_{B^{*}}\right\|_{L_{\rho^{\alpha}}^{q}(\Omega)} .
$$

By (2.16), $\eta_{1}$ and $\eta$ are equivalent in size independent of $\Omega$, and it is now clear that (1.8) holds. This completes the proof of Theorem 1.1.

Remark 3.3. $\quad$ (i) We claim that under the hypotheses of Theorem 1.1, the following Poincaré inequality also holds for both parts (i) and (ii) of the theorem:

$$
\begin{aligned}
& \left\|f-f_{\mathcal{D}}\right\|_{L_{\rho^{\alpha}}^{q}(\Omega)} \leq C \eta^{\frac{\beta}{p}-\frac{\alpha}{q}}|\Omega|^{\frac{1}{q}-\frac{1}{p}} \operatorname{diam}(\Omega)^{1-\frac{\beta}{p}+\frac{\alpha}{q}}\|\nabla f\|_{L_{\rho^{\beta}}^{p}(\Omega)}+ \\
& C \frac{\rho^{\alpha}(\Omega)^{1 / q}}{\min \left\{|\mathcal{D}|,\left|B^{*}\right|\right\}} \operatorname{diam}\left(\mathcal{D}^{\prime}\right)\|\nabla f\|_{L^{1}\left(\mathcal{D}^{\prime}\right)},
\end{aligned}
$$


with $C=C(p, q, \alpha, \beta, n)$, for any convex domain $\mathcal{D} \subset \Omega$ and for all locally Lipschitz continuous functions $f$ on $\Omega$, where $f_{\mathcal{D}}=\int_{\mathcal{D}} f /|\mathcal{D}|, \mathcal{D}^{\prime}$ is any convex domain containing both $\mathcal{D}$ and $B^{*}$, and $B^{*}$ is the central $\Phi$-ball as before. In fact, we have

$$
\left\|f-f_{\mathcal{D}}\right\|_{L_{\rho^{\alpha}}^{q}(\Omega)} \leq\left\|f-f_{B^{*}}\right\|_{L_{\rho^{\alpha}}^{q}(\Omega)}+\rho^{\alpha}(\Omega)^{1 / q}\left|f_{B^{*}}-f_{\mathcal{D}}\right| ;
$$

the first term on the right was estimated in (3.8) and (3.9), and by [CW3, Theorem 1.2],

$$
\begin{aligned}
\left|f_{B^{*}}-f_{\mathcal{D}}\right| \leq & \left|f_{B^{*}}-f_{\mathcal{D}^{\prime}}\right|+\left|f_{\mathcal{D}^{\prime}}-f_{\mathcal{D}}\right| \\
\leq & \frac{1}{\left|B^{*}\right|} \int_{B^{*}}\left(\left|f_{B^{*}}-f\right|+\left|f-f_{\mathcal{D}^{\prime}}\right|\right) \\
& +\frac{1}{|\mathcal{D}|} \int_{\mathcal{D}}\left(\left|f_{\mathcal{D}}-f\right|+\left|f-f_{\mathcal{D}^{\prime}}\right|\right) \\
\leq & \frac{\operatorname{diam}\left(B^{*}\right)}{2\left|B^{*}\right|}\|\nabla f\|_{L^{1}\left(B^{*}\right)}+\frac{\operatorname{diam}\left(\mathcal{D}^{\prime}\right)}{2\left|B^{*}\right|}\|\nabla f\|_{L^{1}\left(\mathcal{D}^{\prime}\right)} \\
& +\frac{\operatorname{diam}(\mathcal{D})}{2|\mathcal{D}|}\|\nabla f\|_{L^{1}(\mathcal{D})}+\frac{\operatorname{diam}\left(\mathcal{D}^{\prime}\right)}{2|\mathcal{D}|}\|\nabla f\|_{L^{1}\left(\mathcal{D}^{\prime}\right)} \\
\leq & 2 \frac{\operatorname{diam}\left(\mathcal{D}^{\prime}\right)}{\min \left\{|\mathcal{D}|,\left|B^{*}\right|\right\}}\|\nabla f\|_{L^{1}\left(\mathcal{D}^{\prime}\right)} .
\end{aligned}
$$

The claim now follows. Also, for any $E \subset \Omega$ of positive measure, Hölder's inequality gives

$$
\begin{aligned}
\rho^{\alpha}(\Omega)^{1 / q}\left|f_{\Omega, \rho^{\alpha}}-f_{E, \rho^{\alpha}}\right| & \leq\left(\frac{\rho^{\alpha}(\Omega)}{\rho^{\alpha}(E)}\right)^{1 / q}\left\|f-f_{\Omega, \rho^{\alpha}}\right\|_{L_{\rho^{\alpha}}^{q}(E)} \\
& \leq\left(\frac{\rho^{\alpha}(\Omega)}{\rho^{\alpha}(E)}\right)^{1 / q}\left\|f-f_{\Omega, \rho^{\alpha}}\right\|_{L_{\rho^{\alpha}}^{q}(\Omega)} .
\end{aligned}
$$

It follows from Theorem 1.1 that

$\left\|f-f_{E, \rho^{\alpha}}\right\|_{L_{\rho^{\alpha}}^{q}(\Omega)} \leq C\left(\frac{\rho^{\alpha}(\Omega)}{\rho^{\alpha}(E)}\right)^{1 / q} \eta^{\frac{\beta}{p}-\frac{\alpha}{q}}|\Omega|^{\frac{1}{q}-\frac{1}{p}} \operatorname{diam}(\Omega)^{1-\frac{\beta}{p}+\frac{\alpha}{q}}\|\nabla f\|_{L_{\rho^{\beta}}^{p}(\Omega)}$, and consequently by arguments similar to those above,

$$
\begin{aligned}
\left\|f-f_{E}\right\|_{L_{\rho^{\alpha}}^{q}(\Omega)} \leq C\left(\frac{\rho^{\alpha}(\Omega)}{\rho^{\alpha}(E)}\right)^{\frac{1}{q}} \eta^{\frac{\beta}{p}-\frac{\alpha}{q}}|\Omega|^{\frac{1}{q}-\frac{1}{p}} \operatorname{diam}(\Omega)^{1-\frac{\beta}{p}+\frac{\alpha}{q}}\|\nabla f\|_{L_{\rho^{\beta}}^{p}(\Omega)} \\
+\frac{\rho^{\alpha}(\Omega)^{\frac{1}{q}}}{\rho^{\alpha}(E)}\left\|f-f_{E}\right\|_{L_{\rho^{\alpha}}^{1}(E)} .
\end{aligned}
$$

In particular, if $E$ is a $\delta$-ball then $\rho$ is essentially constant on $E$, and by using the last two inequalities we obtain that $\left\|f-f_{E}\right\|_{L_{\rho^{\alpha}}^{q}(\Omega)}$ is less than

$$
C\left(\frac{\rho^{\alpha}(\Omega)}{\rho^{\alpha}(E)}\right)^{1 / q} \eta^{\frac{\beta}{p}-\frac{\alpha}{q}}|\Omega|^{\frac{1}{q}-\frac{1}{p}} \operatorname{diam}(\Omega)^{1-\frac{\beta}{p}+\frac{\alpha}{q}}\|\nabla f\|_{L_{\rho^{\beta}}^{p}(\Omega)} .
$$

(ii) Using similar ideas, we can replace $\rho$ by $d_{\Phi}$ in Theorem 1.1, that is, with the same hypotheses, 
$\left\|f-f_{\Omega, d_{\Phi}^{\alpha}}\right\|_{L_{d_{\Phi}^{\alpha}}^{q}(\Omega)} \leq C(p, q, \alpha, \beta, n) \eta^{\frac{\beta}{p}-\frac{\alpha}{q}}|\Omega|^{\frac{1}{q}-\frac{1}{p}} \operatorname{diam}(\Omega)^{1-\frac{\beta}{p}+\frac{\alpha}{q}}\|\nabla f\|_{L_{d_{\Phi}^{\beta}}^{p}(\Omega)}$.

This can be proved by using the fact that if $2 B \subset \Omega$, then $d_{\Phi}(x) \sim d_{\Phi}(y)$ for all $x, y \in B$.

(iii) It has already been shown in [CD] that two necessary conditions for (1.8) in case $q \geq p$ are

$$
\frac{1}{q} \geq \frac{1}{p}-\frac{1}{n} \text { and } 1-\frac{n+\beta}{p}+\frac{n+\alpha}{q} \geq 0 .
$$

Let us now show that in case $1 \leq q<p$, it is necessary that $1-(1+\beta) / p+(1+$ $\alpha) / q>0$. For simplicity, consider $n=2$ and let $\Omega=Q_{0}=(-1,1) \times(0,2)$. For $k \in \mathbb{N}, k>1$, and $i=1, \cdots, 2^{k-1}$, set

$$
R_{i}^{k}=\left((i-1) 2^{-k}, i 2^{-k}\right) \times\left(2^{-k}, 2^{-k+1}\right)
$$

and

$$
L_{i}^{k}=\left(-i 2^{-k},-(i-1) 2^{-k}\right) \times\left(2^{-k}, 2^{-k+1}\right) .
$$

Fix a nonzero polynomial $P$ on $Q=[0,1] \times[0,1]$ that vanishes on $\partial Q$. Define a piecewise polynomial function $g_{k}$ on the strip $[-1 / 2,1 / 2] \times\left[2^{-k}, 2^{-k+1}\right]$ with $g_{k}(-x, y)=-g_{k}(x, y)$ as follows: first define $g_{k}$ on the cube $\left[0,2^{-k}\right] \times$ $\left[2^{-k}, 2^{-k+1}\right]$ by

$$
g_{k}(x, y)=P\left(2^{k} x, 2^{k}\left(y-2^{-k}\right)\right) \text { for }(x, y) \in\left[0,2^{-k}\right] \times\left[2^{-k}, 2^{-k+1}\right],
$$

and then extend $g_{k}$ periodically to other cubes $\left[(i-1) 2^{-k}, i 2^{-k}\right] \times\left[2^{-k}, 2^{-k+1}\right]$ and set $g_{k}=0$ elsewhere in $Q_{0}$. It is easy to see that (with $\rho$ defined relative to $Q_{0}$ )

$\int_{Q_{0}} g_{k}(z) \rho(z)^{\alpha} d z=0 \quad$ and $\left\|g_{k}\right\|_{L_{\rho^{\alpha}}^{q}\left(R_{i}^{k}\right)}^{q}=\left\|g_{k}\right\|_{L_{\rho^{\alpha}}^{q}\left(L_{i}^{k}\right)}^{q} \sim 2^{-k(2+\alpha)}\|P\|_{L^{q}(Q)}^{q}$ and

$$
\left\|\nabla g_{k}\right\|_{L_{\rho^{\beta}}^{p}\left(L_{i}^{k}\right)}^{p}=\left\|\nabla g_{k}\right\|_{L_{\rho^{\beta}}^{p}\left(R_{i}^{k}\right)}^{p} \sim 2^{-k(2+\beta-p)}\|\nabla P\|_{L^{p}(Q)}^{p} .
$$

Now let $f_{N}=\sum_{k=2}^{N+1}\left(2^{k}\right)^{(1+\alpha) / q} g_{k}$, and note that $\int_{Q_{0}} f_{N} \rho^{\alpha} d z=0$ and $\left\|f_{N}\right\|_{L_{\rho^{a}}^{q}\left(Q_{0}\right)} /\left\|\nabla f_{N}\right\|_{L_{\rho^{\beta}}^{p}\left(Q_{0}\right)} \sim \frac{\|P\|_{L^{q}(Q)}}{\|\nabla P\|_{L^{p}(Q)}} N^{1 / q} /\left(\sum_{k=2}^{N+1} 2^{k p\left[1-\frac{1+\beta}{p}+\frac{1+\alpha}{q}\right]}\right)^{1 / p}$. If $1-\frac{1+\beta}{p}+\frac{1+\alpha}{q}=0$, the right side equals

$$
\frac{\|P\|_{L^{q}(Q)}}{\|\nabla P\|_{L^{p}(Q)}} N^{1 / q-1 / p} \rightarrow \infty \quad \text { as } N \rightarrow \infty
$$

since $q<p$, while if $1-\frac{1+\beta}{p}+\frac{1+\alpha}{q}<0$, the right side exceeds a positive constant times a similar factor with $N^{1 / q-1 / p}$ replaced by $N^{1 / q}$. It follows that we must have $1-(1+\beta) / p+(1+\alpha) / q>0$.

(iv) Inequality (1.8) is sharp in the sense that the exponent of $\eta$ is optimal, which was already established in [CD]. 


\section{Appendix}

First, let us establish (2.9). Note that if $t>0$ and $x^{o}+\left(\rho_{o} x / t\right) \in \Omega$, we have $x^{o}-\left(\rho_{o} x /(\nu t)\right) \in \Omega$ by applying (1.3) with $k=1 / \nu$. (2.9) is now clear and hence $d(y, x) \leq \nu d(x, y) \leq n d(x, y)$ for all $x, y$.

We now show that $\eta \leq \eta_{1} \leq 2 \eta$, where $\eta_{1}$ is defined in (2.14). In fact, if $x_{1}, y_{1} \in$ $B(0,1)$ then by $(2.7)$,

$$
\left|x_{1}-y_{1}\right| \leq\left|x_{1}\right|+\left|y_{1}\right| \leq \eta \Phi\left(x_{1}\right)+\eta \Phi\left(y_{1}\right)<\eta+\eta=2 \eta,
$$

and therefore $\eta_{1} \leq 2 \eta$. Also, by (2.8) and (2.11), $\max \{|x|: x \in \partial B(0,1)\}=\eta$, so that $\eta \leq \eta_{1}$.

Next, we recall the classical definition of eccentricity and show that $\eta$ is equivalent to it in size. For an open bounded convex set $\Omega$, let $\tilde{R}(\Omega)$ be the infimum of the radii of all Euclidean balls that enclose $\Omega$, and let $\tilde{r}(\Omega)$ be the supremum of the radii of all Euclidean balls enclosed in $\Omega$. Then $\tilde{r}(\Omega)=\max _{x \in \Omega} \rho(x) \leq \tilde{R}(\Omega)$. The classical definition of the eccentricity of $\Omega$ is

$$
\tilde{\eta}=\tilde{R}(\Omega) / \tilde{r}(\Omega) .
$$

We claim that

$$
\frac{1}{2(1+n)} \eta \leq \tilde{\eta} \leq 2 \eta
$$

By $(2.12), r(\Omega)=\rho_{o}=\operatorname{dist}\left(x^{o}, \partial \Omega\right)$, and thus the open Euclidean ball $D\left(x^{o}, r(\Omega)\right)$ with center $x^{o}$ and radius $r(\Omega)$ is contained in $\Omega$. Hence, $r(\Omega) \leq \tilde{r}(\Omega)$. Also, $\operatorname{diam}(\Omega) / 2 \leq \tilde{R}(\Omega) \leq \operatorname{diam}(\Omega)$, and consequently

$$
\tilde{R}(\Omega) \leq \operatorname{diam}(\Omega)=\eta_{1} r(\Omega) \leq 2 \eta r(\Omega) \leq 2 \eta \tilde{r}(\Omega) .
$$

Thus, $\tilde{\eta}=\tilde{R}(\Omega) / \tilde{r}(\Omega) \leq 2 \eta$. It remains to show that $\eta \leq 2(1+n) \tilde{\eta}$. We first show that

$$
\tilde{r}(\Omega) \leq(1+\nu) r(\Omega) .
$$

To see this, suppose for simplicity that $x^{o}=0$, and let $z$ be the center of an open Euclidean ball of largest radius in $\Omega$, i.e., of radius $\tilde{r}(\Omega)$. If $\alpha=1 / \nu$, then $-\alpha z \in \Omega$ by (1.3). Also, since $x^{o}=0,(2.1)$ implies there is a point $y \in \partial \Omega$ with $|y|=\rho_{o}=$ $r(\Omega)=\Phi(y)$. Since the closure of the Euclidean ball $D(z, \tilde{r}(\Omega))$ lies in $\bar{\Omega}$, so does the point $z+\tilde{r}(\Omega) y^{\prime}$ with $y^{\prime}=y /|y|$. Note that $\Phi\left(y^{\prime}\right)=1$. The two triangles with vertices $-\alpha z, z, z+\tilde{r}(\Omega) y^{\prime}$ and $-\alpha z, 0, \frac{\alpha}{1+\alpha} \tilde{r}(\Omega) y^{\prime}$ are coplanar and similar, and the point $\frac{\alpha}{1+\alpha} \tilde{r}(\Omega) y^{\prime}$ is the intersection of the line segment $\overline{0 y}$ with the one from $\alpha z$ to $z+\tilde{r}(\Omega) y^{\prime}$. Since $\bar{\Omega}$ is convex, this point lies in $\bar{\Omega}$. As $\bar{\Omega}=\{x: \Phi(x) \leq r(\Omega)\}$, we obtain $\Phi\left(\frac{\alpha}{1+\alpha} \tilde{r}(\Omega) y^{\prime}\right) \leq r(\Omega)$ or $\frac{\alpha}{1+\alpha} \tilde{r}(\Omega) \leq r(\Omega)$, which is the same as (4.3). Finally,

$$
\eta=\frac{\max \{|x|: x \in \partial \Omega\}}{r(\Omega)} \leq \frac{\operatorname{diam}(\Omega)}{r(\Omega)} \leq \frac{2 \tilde{R}(\Omega)}{\tilde{r}(\Omega) /(1+\nu)}=2(1+\nu) \tilde{\eta} \leq 2(1+n) \tilde{\eta} .
$$

This completes the proof that our definition of eccentricity is equivalent to the standard one.

Proof of Lemma 2.3. To show part (i), fix $t$ with $0<t \xi<\rho_{o}$. Then $t z^{\prime} \in \Omega$ since $\Phi\left(t z^{\prime}\right)=t \Phi\left(z^{\prime}\right)=t \xi<\rho_{o}$. By $(2.3), \Phi\left(\rho_{o} z^{\prime} / \xi\right)=\rho_{o}$, and so the point 
$\left(\rho_{o} / \xi\right) z^{\prime} \in \partial \Omega$ by $(2.12)$. Hence $d_{\Phi}\left(t z^{\prime}\right) \leq \rho_{o}-t \xi$ since

$$
\begin{gathered}
d_{\Phi}\left(t z^{\prime}\right)=\min \left\{\Phi\left(y-t z^{\prime}\right): y \in \Omega^{c}\right\} \\
\leq \Phi\left(\frac{\rho_{o}}{\xi} z^{\prime}-t z^{\prime}\right)=\left(\frac{\rho_{o}}{\xi}-t\right) \Phi\left(z^{\prime}\right)=\rho_{o}-t \xi .
\end{gathered}
$$

To show the opposite inequality $d_{\Phi}\left(t z^{\prime}\right) \geq \rho_{o}-t \xi$, we first claim that $B\left(t z^{\prime}, \rho_{o}-t \xi\right) \subset$ $\Omega$. In fact, if $w \in B\left(t z^{\prime}, \rho_{o}-t \xi\right)$, then

$$
\Phi(w) \leq \Phi\left(w-t z^{\prime}\right)+\Phi\left(t z^{\prime}\right)<\rho_{o}-t \xi+t \Phi\left(z^{\prime}\right)=\rho_{o},
$$

proving the claim. Now fix any $y \in \partial \Omega$ and let $\tilde{y}$ be the point of intersection of $\partial B\left(t z^{\prime}, \rho_{o}-t \xi\right)$ and the line segment connecting $t z^{\prime}$ and $y$. Then $y-t z^{\prime}=c\left(\tilde{y}-t z^{\prime}\right)$ for some $c \geq 1$, and consequently

$$
\Phi\left(y-t z^{\prime}\right)=c \Phi\left(\tilde{y}-t z^{\prime}\right) \geq \Phi\left(\tilde{y}-t z^{\prime}\right)=\rho_{o}-t \xi .
$$

Thus $d_{\Phi}\left(t z^{\prime}\right) \geq \rho_{o}-t \xi$ and part (i) is proved. Note that part (i) implies that $\left(\rho_{o} / \xi\right) z^{\prime} \in$ $\partial B\left(t z^{\prime}, \rho_{o}-t \xi\right)$.

For part (ii), suppose that $0<t_{1}<t_{0}$. Let us first show that if $t_{0}-t_{1}+r_{1} \leq r_{0}$, then $B\left(t_{1} z^{\prime}, r_{1} \bar{\xi}\right) \subset B\left(t_{0} z^{\prime}, r_{0} \bar{\xi}\right)$. Let $y \in B\left(t_{1} z^{\prime}, r_{1} \bar{\xi}\right)$. Then

$$
\Phi\left(y-t_{0} z^{\prime}\right) \leq \Phi\left(y-t_{1} z^{\prime}\right)+\Phi\left(\left(t_{0}-t_{1}\right)\left(-z^{\prime}\right)\right)<r_{1} \bar{\xi}+\left(t_{0}-t_{1}\right) \bar{\xi} \leq r_{0} \bar{\xi},
$$

so $y \in B\left(t_{0} z^{\prime}, r_{0} \bar{\xi}\right)$ as desired. Conversely, since $B\left(t_{1} z^{\prime}, r_{1} \bar{\xi}\right) \subset B\left(t_{0} z^{\prime}, r_{0} \bar{\xi}\right)$, it follows by taking closures that if $y$ satisfies $\Phi\left(y-t_{1} z^{\prime}\right) \leq r_{1} \bar{\xi}$, then $\Phi\left(y-t_{0} z^{\prime}\right) \leq r_{0} \bar{\xi}$. Choosing $y=\left(t_{1}-r_{1}\right) z^{\prime}$ and noting that $\Phi\left(\left(t_{1}-r_{1}\right) z^{\prime}-t_{1} z^{\prime}\right)=\Phi\left(-r_{1} z^{\prime}\right)=r_{1} \bar{\xi}$, we obtain $\Phi\left(\left(t_{1}-r_{1}-t_{0}\right) z^{\prime}\right) \leq r_{0} \bar{\xi}$, or equivalently, $-\left(t_{1}-r_{1}-t_{0}\right) \bar{\xi} \leq r_{0} \bar{\xi}$. Hence $t_{0}-t_{1}+r_{1} \leq r_{0}$ and (ii) is proved.

We now show (iii). Under the hypothesis of (iii), it follows from (ii) that

$$
t_{0}-t_{1}-(r / \bar{\xi})>r_{0} / \bar{\xi}, \text { and hence } t_{0}-t_{1}>\left(r_{0} / \bar{\xi}\right)+(r / \bar{\xi})>r_{0} / \bar{\xi} \text {. }
$$

By hypothesis and part (i),

$$
r=\delta d_{\Phi}\left(t_{1} z^{\prime}\right)=\delta\left(\rho_{o}-t_{1} \xi\right)>\delta \xi\left(t_{0}-t_{1}\right),
$$

where to obtain the last inequality we used the hypothesis of (iii). Combining estimates gives

$$
r>\delta \xi r_{0} / \bar{\xi} \geq \delta r_{0} / \nu \text { since } \bar{\xi} / \xi \leq \nu \text {. }
$$

Finally, to verify part (iv), let $z \in B\left(x, \delta d_{\Phi}(x)\right) \cap B\left(y, \delta d_{\Phi}(y)\right)$, and choose $\zeta$ with $d_{\Phi}(x)=\Phi(\zeta-x)$. Then

$$
\begin{gathered}
d_{\Phi}(y) \leq \Phi(\zeta-y) \leq \Phi(\zeta-x)+\Phi(x-z)+\Phi(z-y) \\
\leq \Phi(\zeta-x)+\nu \Phi(z-x)+\Phi(z-y) \leq d_{\Phi}(x)+\nu \delta d_{\Phi}(x)+\delta d_{\Phi}(y) .
\end{gathered}
$$

Combining terms, we obtain

$$
d_{\Phi}(y) \leq \frac{1+\nu \delta}{1-\delta} d_{\Phi}(x)<(2+\nu) d_{\Phi}(x) \quad \text { since } \delta<1 / 2 .
$$

This proves the first assertion in part (iv), and the remaining ones are left to the reader, completing the proof of Lemma 2.3 .

Proof of Proposition 2.4. The main idea of the proof is standard but must be adapted to $\Phi$-balls. First, note by Lemma $2.3\left(\right.$ iv) that if $B_{1}$ and $B_{2}$ are two $\Phi$-balls 
whose closures intersect and $r\left(B_{1}\right) \leq 2 r\left(B_{2}\right)$, then $B_{1} \subset(3+2 \nu) B_{2}$. We will write $c_{\nu}=3+2 \nu$.

We will now construct a preliminary cover of $\Omega$ by modifying the Vitali-type argument used in $[\mathrm{Z}]$ and $[\mathrm{CD}]$. We may assume without loss of generality that $x^{o}=0$, so that $\Omega=B\left(0, \rho_{o}\right)$ with $\rho_{o}=\operatorname{dist}(0, \partial \Omega)$. Fix $0<\delta^{\prime}<1 /\left(4 c_{\nu}\right)$ and let $d_{\Phi}$ be as usual. Set $F=\left\{B\left(x, \delta^{\prime} d_{\Phi}(x)\right): x \in \Omega\right\}$ and $R=\sup \{r(B): B \in F\}$. First, let $F_{1}=\{B \in F: R / 2<r(B) \leq R\}$ and choose a maximal pairwise disjoint subfamily $G_{1}$ of $F_{1}$ such that $B\left(0, \delta^{\prime} \rho_{o}\right) \in G_{1}$. Note that $\bigcup_{B \in F_{1}} B \subset \bigcup_{B \in G_{1}} c_{\nu} B$. Then let $F_{2}=\left\{B \in F: R / 4<r(B) \leq R / 2, B \cap \bigcup_{B_{\alpha} \in G_{1}} B=\emptyset\right\}$, and again choose a maximal pairwise disjoint subfamily $G_{2}$ of $F_{2}$. Continuing the process, we find a family $F^{\prime}=\bigcup G_{i} \subset F$ of pairwise disjoint balls such that $\Omega \subset \bigcup_{B^{\prime} \in F^{\prime}} c_{\nu} B^{\prime}$.

Next, fix any $x_{0} \neq 0$ and denote $x_{0} /\left|x_{0}\right|$ by $x_{0}^{\prime}$, so that $x_{0}=\left|x_{0}\right| x_{0}^{\prime}$. We now construct the desired sequence of balls centered along the line segment from $x_{0}$ to 0 . Let $Q_{0}^{\prime}=B\left(x_{0}, \delta^{\prime} d_{\Phi}\left(x_{0}\right)\right)$. The collection of $\delta^{\prime}$-Whitney $\Phi$-balls centered on the segment $\overline{x_{0} 0}$ is $\left\{B\left(t x_{0}^{\prime}, \delta^{\prime} d_{\Phi}\left(t x_{0}^{\prime}\right)\right): 0 \leq t \leq\left|x_{0}\right|\right\}$. For any such ball, we have $t \leq\left|x_{0}\right|=\Phi\left(x_{0}\right) / \Phi\left(x_{0}^{\prime}\right)<\rho_{o} / \Phi\left(x_{0}^{\prime}\right)$, and therefore by Lemma 2.3(i),

$$
d_{\Phi}\left(t x_{0}^{\prime}\right)=\rho_{o}-t \Phi\left(x_{0}^{\prime}\right),
$$

which increases as $t$ decreases. The balls which correspond to $t=0$ and to $t=\left|x_{0}\right|$ are $B\left(0, \delta^{\prime} \rho_{o}\right)$ and $Q_{0}^{\prime}$ respectively. If $B\left(0, \delta^{\prime} \rho_{o}\right)$ and $Q_{0}^{\prime}$ are disjoint, we can choose $t_{1}$ with $0 \leq t_{1}<\left|x_{0}\right|$ such that the ball $Q_{1}^{\prime}=B\left(t_{1} x_{0}^{\prime}, \delta^{\prime} d_{\Phi}\left(t_{1} x_{0}^{\prime}\right)\right)$ is disjoint from $Q_{0}^{\prime}$ but $Q_{0}^{\prime} \subset c_{\nu} Q_{1}^{\prime}$. Here we use the fact that if the closures of $Q_{1}^{\prime}$ and $Q_{0}^{\prime}$ intersect, then $Q_{0}^{\prime} \subset c_{\nu} Q_{1}^{\prime}$ as $r\left(Q_{1}^{\prime}\right)>r\left(Q_{0}^{\prime}\right)$. The increasing nature of $d_{\Phi}\left(t x_{0}^{\prime}\right)$ as $t$ decreases guarantees that we can continue the process a finite number $N-1$ of times (depending on $\left.x_{0}\right)$ until $Q_{N-1}^{\prime}$ intersects $\left.B\left(0, \delta^{\prime} \rho_{o}\right)\right)$, and we let $Q_{N}^{\prime}=B\left(0, \delta^{\prime} \rho_{o}\right)$. The balls $\left\{Q_{i}=c_{\nu} Q_{i}^{\prime}\right\}$ have bounded intercepts depending only on $n$ by a standard volume argument as $\left\{Q_{i}^{\prime}\right\}$ are disjoint, except for the last one $B\left(0, \delta^{\prime} \rho_{o}\right)$, and since intersecting $Q_{i}$ have comparable radii by Lemma 2.3(iv). Since $Q_{i-1}$ intersects $Q_{i}$ and both are $c_{\nu} \delta^{\prime}$-Whitney $\Phi$-balls with $c_{\nu} \delta^{\prime}<1 / 2$, then $Q_{i} \subset C(\nu) Q_{i-1}$ and vice versa by Lemma 2.3(iv). Furthermore, $Q_{i-1}^{\prime} \subset Q_{i} \cap Q_{i-1}$ and $Q_{i} \cup Q_{i-1} \subset C(\nu) Q_{i-1} \subset C(\nu) Q_{i-1}^{\prime}$. Next, let us show that $Q_{0} \subset C\left(\nu, \delta^{\prime}\right) Q_{i}$ for all $i$. Let $w \in Q_{0}=c_{\nu} Q_{0}^{\prime}$. Since $r\left(Q_{0}^{\prime}\right)=\delta^{\prime}\left(\rho_{o}-\left|x_{0}\right| \Phi\left(x_{0}^{\prime}\right)\right)$, then

$$
\begin{gathered}
\Phi\left(w-t_{i} x_{0}^{\prime}\right) \leq \Phi\left(w-x_{0}\right)+\Phi\left(x_{0}-t_{i} x_{0}^{\prime}\right) \\
\leq c_{\nu} \delta^{\prime}\left(\rho_{o}-\left|x_{0}\right| \Phi\left(x_{0}^{\prime}\right)\right)+\left(\left|x_{0}\right|-t_{i}\right) \Phi\left(x_{0}^{\prime}\right) \\
\leq c_{\nu} \delta^{\prime}\left(\rho_{o}-t_{i} \Phi\left(x_{0}^{\prime}\right)\right)+\left(\Phi\left(x_{0}\right)-t_{i} \Phi\left(x_{0}^{\prime}\right)\right) \leq\left(c_{\nu} \delta^{\prime}+1\right)\left(\rho_{o}-t_{i} \Phi\left(x_{0}^{\prime}\right)\right) .
\end{gathered}
$$

Since $r\left(Q_{i}\right)=c_{\nu} \delta^{\prime}\left(\rho_{o}-t_{i} \Phi\left(x_{0}^{\prime}\right)\right)$, it follows that $w \in C\left(\nu, \delta^{\prime}\right) Q_{i}$ with $C\left(\nu, \delta^{\prime}\right)=$ $\left(c_{\nu} \delta^{\prime}+1\right) /\left(c_{\nu} \delta^{\prime}\right)$, as desired.

We now construct a Boman cover of $\Omega$. Let $0<\delta<1 / 2$. For $c_{\nu}$ as above and a suitably large constant $C(\nu)$ to be chosen, let $\bar{c}_{\nu}=C(\nu) c_{\nu}$ and choose $\delta^{\prime}$ with $\bar{c}_{\nu} \delta^{\prime}=\delta$. Use $\delta^{\prime}$ to construct the collection $F^{\prime}$ of pairwise disjoint $\delta^{\prime}$-Whitney $\Phi$-balls exactly as above. Our Boman cover will consist of the $\delta$-Whitney $\Phi$-balls $\left\{\bar{c}_{\nu} B^{\prime}: B^{\prime} \in F^{\prime}\right\}$. Fix $B_{0}^{\prime}=B\left(x_{0}, r_{0}\right) \in \mathcal{F}^{\prime}, x_{0} \neq 0$. Let $\left\{Q_{0}, Q_{1}, \cdots, Q_{N}=B\left(0, c_{\nu} \delta^{\prime} \rho_{o}\right)\right\}$ be the sequence of $c_{\nu} \delta^{\prime}$-Whitney $\Phi$-balls centered on the segment $\overline{x_{0} 0}$ as above. For $i=1, \ldots, N$, choose $B_{i}^{\prime} \in F^{\prime}$ so that $c_{\nu} B_{i}^{\prime}$ contains the center of $Q_{i}$. Of course, we will always choose $c_{\nu} B_{N}^{\prime}=Q_{N}=B\left(0, c_{\nu} \delta^{\prime} \rho_{o}\right)$. Since $c_{\nu} B_{i}^{\prime}$ and $Q_{i}$ are intersecting $c_{\nu} \delta^{\prime}$-Whitney balls with $c_{\nu} \delta^{\prime}<1 / 2$, Lemma 2.3(iv) shows there exists $C(\nu)$ such that $Q_{i} \subset \bar{c}_{\nu} B_{i}^{\prime}$, 
where $\bar{c}_{\nu}=C(\nu) c_{\nu}$. Similarly, $Q_{i-1} \subset \bar{c}_{\nu} B_{i-1}^{\prime}$. Since $Q_{i-1}^{\prime} \subset Q_{i} \cap Q_{i-1}$, then $Q_{i-1}^{\prime} \subset \bar{c}_{\nu} B_{i}^{\prime} \cap \bar{c}_{\nu} B_{i-1}^{\prime}$, and since $\bar{c}_{\nu} B_{i}^{\prime}, \bar{c}_{\nu} B_{i-1}^{\prime}, \bar{c}_{\nu} Q_{i-1}^{\prime}$ are intersecting $\bar{c}_{\nu} \delta^{\prime}$-balls, we have $\bar{c}_{\nu} B_{i}^{\prime} \cup \bar{c}_{\nu} B_{i_{1}}^{\prime} \subset C(\nu) \bar{c}_{\nu} Q_{i-1}^{\prime}$. In particular,

$$
Q_{i-1}^{\prime} \subset \bar{c}_{\nu} B_{i}^{\prime} \cap \bar{c}_{\nu} B_{i-1}^{\prime} \subset C(\nu) \bar{c}_{\nu} Q_{i-1}^{\prime} .
$$

It is now easy to see that $W=\left\{\bar{c}_{\nu} B^{\prime}: B^{\prime} \in F^{\prime}\right\}$ is a Boman cover with $M_{1}$ depending only on $n$ and $M_{2}$ depending only on $\delta, n$. Note that balls in $W$ have bounded intercepts (depending on $n$ ) again by a volume argument, using the fact that intersecting Whitney balls have comparable radii and $\left\{B / \bar{c}_{\nu}: B \in W\right\}$ are pairwise disjoint. This completes the proof of Proposition 2.4 .

\section{References}

[AD] G. Acosta and R. G. Durán, An optimal Poincaré inequality in $L^{1}$ for convex domains, Proc. Amer. Math. Soc. 132 (2004), 195-202.

[BM95] L. P. Boss and P. D. Milman, Sobolev-Gagliardo-Nirenberg and Markov type inequalities on subanalytic domains, Geometric and Functional Analysis 5 (1995), 853-923.

[BKL] S. Buckley, P. Koskela and G. Lu, Boman equals John, Proceedings of the 16th Rolf Nevanlinna Colloquium, 1995, 91-99.

[C93] Seng-Kee Chua, Weighted Sobolev's inequalities on domains satisfying the chain condition, Proc. Amer. Math. Soc. 117 (1993), no.2, 449-457.

[C95] Seng-Kee Chua, Weighted Sobolev interpolation inequalities on certain domains, J. London Math. Soc. (2) 51 (1995), 532-544.

[C01] Seng-Kee Chua, Weighted inequalities on John domains, J. Math. Analysis Appl. 258 (2001), 763-776.

[C09] Seng-Kee Chua, Sobolev interpolation inequalities on generalized John domains, Pacific J. Math. 242 (2009), 215-258.

[CD] Seng-Kee Chua and Huo-Yuan Duan, Weighted Poincaré equalities on symmetric convex domains, Indiana Math. J. 58 (2009), 2103-2114.

[CW1] Seng-Kee Chua and R. L. Wheeden, Sharp conditions for weighted 1-dimensional Poincaré inequalities, Indiana Math. J. 49 (2000), 143-175.

[CW2] Seng-Kee Chua and R. L. Wheeden, A note on sharp 1-dimensional Poincaré inequalities, Proc. Amer. Math. Soc. 134 (2006), 2309-2316.

[CW3] Seng-Kee Chua and R. L. Wheeden, Estimates of best constants for weighted Poincaré inequalities on convex domains, Proc. London Math. Soc. 93 (2006), 197-226.

[CWa] Seng-Kee Chua and R. L. Wheeden, Self-improving properties of Poincaré-type inequalities on general measure spaces and their applications, J. Functional Analysis 255 (2008), 2977-3007.

[CWb] Seng-Kee Chua and R. L. Wheeden, Self-Improving properties of Poincaré-type inequalities on $s$-John domains, Pacific J. Math., to appear.

[CW6] Seng-Kee Chua and R. L. Wheeden, Weighted inequalities of Poincaré type on Boman type domains, preprint.

[CoW] R. Coifman and G. Weiss, Analyse harmonique non-commutative sur certains espaces homogénes, Lecture Notes in Math. 242, Springer-Verlag, New York, 1971.

[DL] S. Dekel and D. Leviatan, The Bramble-Hilbert lemma for convex domains, Siam J. Math. Analysis 35 (2004), 1203-1212.

[FPW1] B. Franchi, C. Pérez and R. L. Wheeden, Self-improving properties of John-Nirenberg and Poincaré inequalities on spaces of homogeneous type, J. Functional Analysis 153 (1998), 108-146.

[FPW2] B. Franchi, C. Pérez and R. L. Wheeden, A sum operator with applications to self-improving properties of Poincaré inequalities in metric spaces, J. Fourier Analysis Appl. 9 (2003), 511-540.

[G] J. Gardner, The Brunn-Minkowski inequality, Bull. Amer. Math. Soc. 39 (2002), 355-405.

[HS] Ritva Hurri-Syrjanen, An improved Poincaré inequality, Proc. Amer. Math. Soc. 120 (1994), 213-222.

[L] P. Lax, Functional Analysis, Wiley-Interscience, 2002.

[W] R. Webster, Convexity, Oxford University Press, 1994. 
[Wh] R. L. Wheeden, A characterization of some weighted norm inequalities for the fractional maximal function, Studia Math. 107 (1993), 257-272.

[Z] William P. Ziemer, Weakly Differentiable Functions, Springer Verlag, New York 1989.

Department of Mathematics, 10, Lower Kent Ridge Rd., National University of SingaPORE, Singapore 119076

E-mail address: matcsk@nus.edu.sg

Department of Mathematics, Rutgers University, Piscataway, NJ 08854

E-mail address: wheeden@math.rutgers.edu 